\title{
Risques professionnels et grossesse dans les métiers de la coiffure et de l'esthétique : enquête auprès des médecins du travail sur leur pratique
}

\author{
Professional risks and pregnancy for hairdressers and \\ estheticians: An inquiry about their practice with occupational \\ physicians

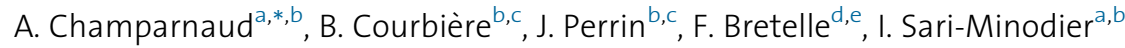
a Service hospitalo-universitaire de médecine et santé au travail, faculté de médecine, Assistance publique-Hôpitaux de Marseille (APHM), Aix-Marseille université, 27, boulevard Jean-Moulin, 13385 Marseille cedex 5, France
${ }^{\mathrm{b}}$ IMBE UMR 7263, Aix-Marseille université, CNRS, IRD, université d'Avignon, 13013 Marseille, France
${ }^{\mathrm{C} C e n t r e ~ c l i n i c o-b i o l o g i q u e ~ d ' a s s i s t a n c e ~ m e ́ d i c a l e ~ a ̀ ~ l a ~ p r o c r e ́ a t i o n, ~ p o ̂ l e ~ f e m m e s ~ p a r e n t s ~}$ enfants, Assistance publique-Hôpitaux de Marseille (APHM) la Conception, 13005 Marseille, France
${ }^{\mathrm{d}}$ Service de gynécologie-obstétrique, pôle femmes parents enfants, Assistance publique- Hôpitaux de Marseille (APHM), hôpital Nord, 13015 Marseille, France
e UM63, CNRS 7278, IRD 198, Inserm 1095, unité de recherche sur les maladies infectieuses tropicales et émergentes, Aix-Marseille université, 13005 Marseille, France

\section{Summary}

Purpose of the study. The branch of cosmetology, employing women in majority, is exposed to numerous professional risks. Therefore prevention of potential risks for reproduction has to be considered. We have led an inquiry from occupational physicians in order to know more about their practice, their resources and the difficulties they meet in the prevention approach used for pregnant or old enough women to procreate.

Methods. A specific questionnaire has been developed and the occupational physicians in PACA area were invited to answer online, through the mailing list of the regional occupational medicine and health society (257 doctors).

Results. Among the ninety-two doctors who gave an answer, fortysix declared they were in charge of the medical surveillance of hairdressers, estheticians and/or nail technicians. Only eight of them said they happened to have to face questions related to pregnancy for employees in this branch. However, all the doctors pointed out their difficulties when trying to identify the components of cosmetic

\section{Résumé}

Objectif. Le secteur de la cosmétique, employant très majoritairement des femmes, expose à de nombreux risques professionnels. La prévention des risques potentiels pour la reproduction est donc un enjeu important. Nous avons conduit une enquête auprès des médecins du travail, en charge de salons de coiffure ou d'esthétique, afin de mieux connaître leurs pratiques, leurs ressources et leurs difficultés dans la démarche de prévention vis-à-vis des femmes en âge de procréer ou enceintes.

Méthode. Un questionnaire spécifique a été construit et les médecins du travail de la région PACA ont été invités à y répondre en ligne, via la liste de diffusion de la société régionale de médecine et santé au travail comptant 257 médecins.

Résultats. Parmi les 92 médecins ayant répondu, 46 ont déclaré être en charge du suivi de coiffeuses, esthéticiennes et/ou prothésistes ongulaires. Parmi ceux-ci, huit seulement ont déclaré avoir été confrontés à des questions en rapport avec la grossesse chez les salariées de ce secteur. Cependant, l'ensemble des médecins ont mis

* Auteur correspondant.

e-mail : amelie.champarnaud@gmail.com (A. Champarnaud). 
products and to assess their reprotoxic risk. Moreover, they identified postural constraints with a serious professional risk for these workers. Discussion and conclusion. It appears that professional risk prevention (information, monitoring, protective equipment), especially regarding pregnancy, could be improved. Measures to protect pregnant women at work (adaptation of the workplace, temporary redeployment to another position, suspension of the employment contract with guarantee of full pay) seem to be rarely implemented in the branch of cosmetology. Furthermore, the knowledge of professional exposure risks for reproduction in those activities is still inadequate. Novel studies seem to be necessary in order to supplement the present data.

Keywords: Pregnancy, Professional risk, Barbering, Esthetics, Occupational health physicians

\section{Introduction}

Concilier travail et grossesse, dans des conditions favorables au bon déroulement de cette dernière, concerne la majorité des femmes enceintes. En effet, en 2010, d'après l'Enquête nationale périnatale (ENP) [1], 70,2 \% des femmes de métropole ont exercé un emploi durant leur grossesse.

De plus, l'enquête SUMER 2010 [2] a estimé à 146700 l'effectif de professionnels de la coiffure et de l'esthétique, avec un taux de féminisation très élevé et en augmentation, soit $95,3 \%$ (85 \% en 2002, $71 \%$ en 1982) [3]. Il s'agit pour la plupart de femmes en âge de procréer : 35,1\% ont moins de 25 ans, 37,9 \% entre 25 et 39 ans et $16,1 \%$ entre 40 et 49 ans. Par ailleurs, toujours d'après SUMER [2], la proportion de salariés de la famille " coiffeurs, esthéticiens " exposés à certains risques professionnels est bien supérieure à celle de l'ensemble des familles professionnelles. C'est le cas notamment de l'exposition aux nuisances chimiques puisque $88,3 \%$ des coiffeurs et esthéticiens y seraient exposés (contre 33,2\% pour l'ensemble des familles professionnelles). Les principaux agents chimiques relevés sont les persulfates de sodium, potassium et ammonium, l'eau oxygénée, l'ammoniac ou les tensioactifs mais la liste de ces produits est longue [2].

Cette étude met également en avant l'exposition aux contraintes posturales et articulaires (position debout prolongée, piétinement, gestes répétitifs, maintien des bras en l'air, position fixe de la tête et du cou, travail exigeant une position forcée). En effet, 96,9\% des coiffeurs et esthéticiens sont soumis à ces types de contraintes, contre $74,3 \%$ pour l'ensemble des familles professionnelles [2]. en avant les difficultés rencontrées dans l'identification des composants des produits cosmétiques et l'évaluation du risque reprotoxique. Ils ont également identifié les contraintes posturales comme risque professionnel majeur chez ces salariées.

Discussion et conclusion. Il apparaît que la prévention des risques professionnels (information, formation, équipements de protection...), notamment en lien avec la grossesse, pourrait être améliorée. Les dispositions relatives à la protection des femmes enceintes au travail (aménagement de poste, changement temporaire d'affectation, suspension du contrat de travail avec garantie de rémunération...) semblent ne pas être fréquemment mises en œuvre dans le secteur de la cosmétique. Par ailleurs, les connaissances sur les risques des expositions professionnelles pour la reproduction dans ces métiers sont encore insuffisantes. De nouvelles études semblent donc nécessaires afin de compléter les données actuelles.

Mots clés : Grossesse, Risques professionnels, Coiffure, Esthétique, Médecins du travail

D'autres contraintes sont également à prendre en compte dans ces professions telles que les aspects psycho-organisationnels (travail le week-end, horaires prolongés, contact avec le public, objectifs chiffrés...), la charge mentale, le risque biologique, la manutention manuelle de charges, les vibrations (prothésistes ongulaires), les rayonnements (UV) ainsi que les autres facteurs physiques et risques liés aux outils de travail (nuisances sonores et thermiques, risque électrique, éclairage, coupure, brûlure. . .) [2,4,5-10].

De nombreuses études ont été menées sur les effets de l'exposition aux risques professionnels (agents chimiques notamment les solvants, troubles musculosquelettiques [TMS], bruit...) chez les femmes enceintes, quelle que soit leur profession [11] et plusieurs ont porté sur le secteur de la cosmétique, du fait de ses risques professionnels et de ses caractéristiques démographiques (nombreuses femmes jeunes). Il s'agit principalement de recherches menées aux ÉtatsUnis ou dans les pays scandinaves et portant sur les " cosmétologistes ", terme regroupant les professions de coiffeuse, esthéticienne, prothésiste ongulaire... Ces études ont fait l'objet de deux méta-analyses récentes.

La première $[12,13]$, conduite par l'Institut national de recherche et de sécurité (INRS), sur la base de 57 publications étudiant divers troubles de la reproduction, a retenu 32 études pour réaliser des méta-analyses sur six types d'effets. Les résultats montrent une augmentation faible, mais statistiquement significative, du délai nécessaire à concevoir (odds ratio $[\mathrm{OR}]: 1,11$, intervalle de confiance à $95 \%[1,03-1,19])$, de la mortalité embryonnaire et fœtale (OR : 1,19 [1,03-1,38]), du retard de croissance intra-utérin (OR : 1,24 [1,10-1,41]) et du 
faible poids de naissance (OR : 1,21 [1,06-1,39]) chez les professionnelles de la coiffure et des soins de beauté. Les résultats sont non significatifs pour les autres effets étudiés (prématurité, malformations congénitales).

La deuxième méta-analyse [14] porte sur 19 études, dont 17 qui avaient été incluses dans la méta-analyse de l'INRS [12]. Elle montre une augmentation faible, statistiquement significative, de la mortalité embryonnaire et fœtale (OR : 1,14 [1,041,24]) et de l'infertilité (OR : 1,15 [1,03-1,28]). Les autres troubles étudiés (retard de croissance intra-utérin, faible poids de naissance, prématurité) ne présentent pas de résultats significatifs.

Il faut noter que les études menées chez les salariées du secteur de la cosmétique ont porté sur l'effet global de la "profession ", soit un effet cumulé de l'ensemble des risques professionnels sur les troubles de la reproduction. Nous n'avons trouvé aucun article évaluant, dans ces métiers de la coiffure et de l'esthétique, l'impact sur la reproduction d'un risque ou classe de risque. De plus, à notre connaissance, aucune étude concernant les troubles de la reproduction n'a été menée de façon spécifique sur la profession d'esthéticienne ou de prothésiste ongulaire.

Face à ces excès de risques, faibles mais significatifs, pour la fertilité et la grossesse dans ces professions, et face à la multiplicité des facteurs de risque présents, dont les effets sur la reproduction sont mal connus, les médecins du travail sont confrontés à divers questionnements. De plus, l'évaluation du risque chimique dans ce secteur se heurte à des difficultés spécifiques, liées au fait que les produits cosmétiques ne sont pas soumis au règlement européen Classification Labelling Packaging (CLP) et ne sont donc pas étiquetés conformément à ce dernier. Ils ne sont pas non plus concernés par l'obligation de fiches de donnees de sécurité (FDS). Enfin, quel que soit le secteur d'activité, les médecins du travail sont souvent interpellés pour des femmes enceintes, dont la grossesse est déjà bien avancée, alors que la démarche d'évaluation et de prévention des risques reprotoxiques devrait être conduite en amont pour permettre, si nécessaire, la mise en œuvre d'actions préventives adaptées, dès la déclaration de grossesse, cette déclaration devant être la plus précoce possible.

Malgré les problématiques qui peuvent se poser aux médecins du travail en charge de salariés du secteur de la cosmétique, nous n'avons pas retrouvé d'étude concernant leur pratique professionnelle vis-à-vis du risque reprotoxique dans ce secteur.

Nous avons donc conduit une enquête auprès des médecins du travail de la région Provence-Alpes-Côte d'Azur (PACA) en charge de salons de coiffure ou d'esthétique afin de mieux connaître leur pratique, leurs ressources et leurs difficultés dans la démarche de prévention vis-à-vis des femmes enceintes et en âge de procréer.

\section{Matériel et méthodes}

\section{Élaboration du questionnaire}

L'enquête est basée sur un auto-questionnaire anonyme, élaboré spécifiquement pour l'étude. Il est composé de 42 questions. Les questions, en majorité à réponses fermées (seules sept questions à réponses ouvertes permettant d'apporter des précisions selon le type d'entreprise ou sur un point particulier) sont regroupées en quatre parties :

- les informations dont les médecins du travail disposent dans les entreprises du secteur de la cosmétique ;

- les autres sources d'information des médecins du travail ;

- la protection et la formation des salariées mises en place par l'employeur ;

- le rôle de l'équipe pluridisciplinaire de santé au travail (actions en milieu de travail et suivi médical).

Les modalités de réponse étaient : "Toujours ", "Souvent ", "Parfois ", " Jamais ", " Ne se prononce pas/Ne sait pas (NSPP/NSP) ", " Non concerné ".

Le questionnaire a été mis en forme puis mis en ligne avec le logiciel Google Sheets ${ }^{\circledR}$. Il a d'abord été validé auprès de six médecins du travail ce qui a permis de clarifier certains items.

\section{Population d'étude, modalités de recrutement, de recueil et d'analyse des données}

L'enquête a été conduite auprès des médecins du travail adhérant à la Société de médecine et santé au travail, d'ergonomie et de toxicologie (SMST) de la région PACA et assurant le suivi de salariées du secteur de la cosmétique.

Une présentation du projet a été faite lors d'une session de la SMST.

Une invitation à participer, comprenant les objectifs de l'enquête, le lien vers le questionnaire, la mention de l'anonymat des réponses et les coordonnées de l'investigateur, a ensuite été adressée par mail via la liste de diffusion de la SMST, comptant alors 257 médecins. Tous les médecins, quels que soient leur mode d'exercice et secteurs d'activité, ont été sollicités, les données de la liste de diffusion ne permettant de faire aucune sélection. Seuls les médecins ayant répondu prendre en charge des coiffeuses, esthéticiennes et/ou prothésistes ongulaires ont été invités à répondre au questionnaire proprement dit.

Le recueil des données s'est déroulé en mai et juin 2016. Une relance sur toute la liste de diffusion de la SMST a été faite par mail quatre semaines a près la première sollicitation. Devant le nombre élevé de non-réponses à la fin du délai de réponse (initialement fixé à 5 semaines), ce dernier a été allongé de deux semaines.

Les réponses au questionnaire en ligne ont été recueillies, de façon anonyme, via le logiciel Google Sheets ${ }^{\circledR}$, puis importées automatiquement dans un tableur Excel ${ }^{\circledR}$. L'analyse 
descriptive des données (effectifs, pourcentages, moyennes et écart-types) a été réalisée à l'aide du logiciel Excel ${ }^{\circledR}$.

\section{Résultats}

Au total, 92 médecins du travail sur les 257 sollicités ont répondu au questionnaire en ligne soit un taux de réponse de $35,8 \%$. Parmi ceux-ci, 46 ont répondu être concernés par le suivi de coiffeuses, esthéticiennes et/ou prothésistes ongulaires et ont donc répondu à la suite du questionnaire.

L'ancienneté d'exercice en médecine du travail est en moyenne de 16,6 $\pm 10,2$ ans, variant de 2 à 35 ans.

Les médecins ont répondu suivre approximativement entre 2 et 100 salariées du secteur de la cosmétique (moyenne de 22 salariées par médecin). Cet effectif se répartit environ en $80 \%$ de salariées en salons indépendants et $20 \%$ en enseignes nationales.

À la question " Avez-vous été confronté à des questions en rapport avec la grossesse au travail dans les professions de la cosmétique ? " posée en début de questionnaire, 8 médecins $(17,4 \%)$ ont répondu de façon positive, $36(78,3 \%)$ ont répondu de façon négative et $2(4,3 \%)$ ont répondu " Ne sait pas ». Au total, 16 médecins (dont 8 avaient pourtant répondu ne jamais avoir été confrontés à des questions en rapport avec la grossesse au travail dans ces professions) ont signalé les problématiques suivantes à l'occasion d'une question ouverte :

- contraintes posturales (signalées par neuf médecins) et notamment station debout prolongée, postures pénibles et gestes répétitifs (dont un cas d'esthéticienne rapportant des contractions en début de grossesse lors de la pratique d'un massage) ;

- exposition aux produits chimiques (rapportée par cing médecins), notamment pour les solvants, l'activité en onglerie et les huiles essentielles en cosmétique ;

- durée de travail (plus de 8 heures par jour);

- temps de transport domicile-travail.

Trois autres médecins ont également pointé, en réponse à la même question ouverte, l'absence d'information du médecin du travail par rapport à la déclaration de grossesse, les visites médicales uniquement de reprise après maternité ainsi que la difficulté à aménager le poste de travail dans le secteur (nécessité de haut rendement) se traduisant par des arrêts maladie fréquents ou une inaptitude temporaire.

La première partie du questionnaire portait sur les informations dont disposent les médecins du travail dans les entreprises du secteur de la cosmétique.

Concernant les déclarations de grossesse, 13 médecins ( $28,3 \%$ ) ont répondu en être informés (souvent ou parfois) par l'employeur.

La plupart des médecins (38 soit 82,6\%) déclaraient rencontrer des difficultés dans l'évaluation des expositions au poste de travail.

Quant au document unique d'évaluation des risques (DUER), 26 médecins $(56,6 \%)$ ont répondu qu'il était mis en place (toujours, souvent ou parfois) dans les entreprises du secteur de la cosmétique (Tableau 1).

Les difficultés le plus souvent rapportées par les médecins (plus de $70 \%$ ) sont l'absence de fiches individuelles d'exposition et la non-communication des FDS (quand elles existent) (Tableau 2). L'absence d'étiquetage des produits et le manque de temps pour les visites d'entreprise sont évoqués par environ la moitié des médecins.

Dans une question ouverte sur les éventuelles différences entre types d'entreprises (enseignes nationales ou salons indépendants), un médecin rapporte que les difficultés dans l'évaluation des expositions concernent surtout les salons indépendants alors qu'un autre note que ces difficultés sont d'avantage présentes dans les enseignes nationales (" peu compliants, DUER global national ne collant pas avec la réalité locale, règles non négociables... alors que dans les petits salons, l'employeur travaille avec ses salariés et comprend l'intérêt d'une prévention qui le concerne aussi "). Deux médecins font état de DUER plus souvent rédigé dans les enseignes nationales. Un autre médecin rapporte qu'un des salons a effectué le DUER à l'aide d'un cabinet indépendant. La deuxième partie du questionnaire s'intéressait aux autres sources d'informations des médecins du travail.

Le Tableau 3 indique les acteurs avec lesquels les médecins répondants ont déjà échangé concernant le risque reprotoxique. Les collègues médecins du travail et les intervenants en prévention des risques professionnels (IPRP) sont les acteurs les plus souvent cités, puis le centre d'information médicale de I'INRS.

\section{Tableau 1}

Réponse des médecins du travail sur les informations dont ils disposent dans l'entreprise : déclarations de grossesse transmises par l'employeur ; difficultés rencontrées par le médecin dans l'évaluation des expositions au poste de travail ; mise en place du document unique d'évaluation des risques (DUER).

\begin{tabular}{|c|c|c|c|c|c|c|}
\hline & Toujours & Souvent & Parfois & Jamais & NSPP/NSP & Total \\
\hline $\begin{array}{l}\text { Déclarations de grossesses transmises } \\
\text { par l'employeur }\end{array}$ & o (o \%) & $5(10,9 \%)$ & $8(17,4 \%)$ & $27(58,7 \%)$ & $6(13 \%)$ & $46(100 \%)$ \\
\hline $\begin{array}{l}\text { Difficultés rencontrées par le médecin dans } \\
\text { l'évaluation des expositions }\end{array}$ & $4(8,7 \%)$ & $15(32,6 \%)$ & $19(41,3 \%)$ & $4(8,7 \%)$ & $4(8,7 \%)$ & $46(100 \%)$ \\
\hline Mise en place du DUER & $1(2,2 \%)$ & $4(8,7 \%)$ & $21(45,7 \%)$ & $18(39,1 \%)$ & $2(4,3 \%)$ & $46(100 \%)$ \\
\hline
\end{tabular}


Tableau 2

Les difficultés rencontrées par les médecins du travail dans l'évaluation des risques dans le secteur de la cosmétique.

Quelles difficultés rencontrez-vous ? (plusieurs réponses possibles parmi les suivantes)

Nombre de réponses (\%)

Absence de communication des fiches de données de sécurité existantes $35(76,1 \%)$

(notamment pour les produits de nettoyage)

Fiches individuelles d'exposition inexistantes

$34(73,9 \%)$

Non étiquetage des produits cosmétiques

$25(54,5 \%)$

Manque de temps pour effectuer les visites d'entreprise et les études de poste

$21(45,7 \%)$

Difficultés pour la rédaction et la mise à jour des fiches d'entreprise

Utilisation de produits cosmétiques hors circuit officiel

$11(23,9 \%)$

Autres

$6(13 \%)$

o (o \%)

\section{Tableau 3}

Acteurs de prévention avec lesquels les médecins du travail ont déjà travaillé ou échangé.

\begin{tabular}{ll}
$\begin{array}{l}\text { Concernant le risque reprotoxique } \\
\text { dans les professions de la } \\
\text { cosmétique, avez-vous déjà } \\
\text { travaillé ou échangé avec l'un ou } \\
\text { plusieurs des acteurs suivants ? }\end{array}$ & $\begin{array}{l}\text { Nombre de } \\
\text { réponses (\%) }\end{array}$ \\
\hline $\begin{array}{l}\text { Aucun } \\
\text { Autres médecins du travail } \\
\text { Intervenant en prévention } \\
\text { des risques professionnels }\end{array}$ & $19(41,3 \%)$ \\
$\begin{array}{l}\text { Centre d'information } \\
\text { médicale de l'INRS }\end{array}$ & $18(39,1 \%)$ \\
$\begin{array}{l}\text { Centre anti-poison et } \\
\text { de toxico-vigilance }\end{array}$ & $15(32,6 \%)$ \\
$\begin{array}{l}\text { Centre de consultation de } \\
\text { pathologie professionnelle }\end{array}$ & $10(21,7 \%)$ \\
$\begin{array}{l}\text { CARSAT } \\
\text { Centre de référence des } \\
\text { agents tératogènes }\end{array}$ & $3(6,5 \%)$ \\
Autres & $2(4,3 \%)$ \\
\hline
\end{tabular}

${ }^{a}$ Un médecin cite les représentants commerciaux des produits.
Le Tableau 4 décrit les documents que les médecins du travail sont amenés à consulter pour guider leur conduite vis-à-vis du risque reprotoxique et de la grossesse au travail. Plus de $75 \%$ des médecins citent la classification réglementaire "Cancérogènes Mutagènes Reprotoxiques " (CMR) [15], plus de $30 \%$ les recommandations de la Haute Autorité de santé (HAS) ou de la Société française de médecine du travail (SFMT) [16-18] et plus de $25 \%$ l'avis d'experts de l'INRS [19].

Les médecins du travail peuvent également être en contact avec les professionnels de santé en charge du suivi de la grossesse, notamment en cas de problème au poste de travail : médecin généraliste pour 37 répondants $(80,5 \%)$, gynécologue pour $35(76,1 \%)$ et sage-femme pour $3(6,5 \%)$ (Tableau 5).

La troisième partie du questionnaire portait sur la protection et la formation des salariées.

Parmi les médecins interrogés, 39 (84,8\%) ont dit suivre des salariées travaillant dans des locaux disposant (toujours, souvent ou parfois) d'équipements de protection collective (EPC) et/ou de matériel ergonomique. Trois médecins indiquent que les entreprises qu'ils suivent n'en disposent jamais. La liste des EPC en fonction de la fréquence des réponses des médecins est présentée dans le Tableau 6.

\section{Tableau 4}

Documents consultés par les médecins pour guider la conduite à tenir vis-à-vis du risque reprotoxique et de la grossesse au travail.

Quels sont les documents que vous êtes amenés à consulter pour guider votre conduite vis-à-vis

du risque reprotoxique et de la grossesse au travail (en général, pour tous les secteurs d'activité, et pas seulement celui de la cosmétique) ? (plusieurs réponses possibles)

Classification réglementaire "Cancérogènes mutagènes reprotoxiques " (CMR) [15]

Recommandations de la Société française de médecine du travail : "Salariées enceintes exposées à des substances toxiques pour le développement fœtal. Surveillance médicale. " (2004) [16]

Recommandations de la Haute Autorité de santé : "Comment mieux informer les femmes enceintes ? Recommandations pour les professionnels de santé (2005) [17] et/ou

"Suivi et orientation des femmes enceintes en fonction des situations à risque identifiées. " (2007) [18]

Avis d'experts : "Grossesse et travail. Quels sont les risques pour l'enfant à naître ? " (INRS, 2010) [19]

Fiches DEMETER [20]

Autres

Aucun

Nombre de réponses (\%)

${ }^{a}$ Un médecin fait référence à des sites Internet spécialisés, un à des documents internes au service de santé au travail et un aux fiches EVARIST (logiciel de partage de " fiches substances "). 
Tableau 5

Professionnels de santé en charge du suivi de la grossesse avec lesquels les médecins du travail sont en lien.

\begin{tabular}{|c|c|c|c|c|c|}
\hline $\begin{array}{l}\text { Ėtes-vous en lien avec les professionnels de santé en } \\
\text { charge du suivi de la grossesse en cas de problème au } \\
\text { poste de travail (en général, pour tous les secteurs } \\
\text { d'activité, et pas seulement celui de la cosmétique) ? }\end{array}$ & Toujours & Souvent & Parfois & Jamais & Total \\
\hline Médecin généraliste & $4(8,7 \%)$ & $9(19,6 \%)$ & $24(52,2 \%)$ & $9(19,6 \%)$ & $46(100 \%)$ \\
\hline Gynécologue & $1(2,2 \%)$ & $9(19,6 \%)$ & $25(54,3 \%)$ & $11(23,9 \%)$ & $46(100 \%)$ \\
\hline Sage-femme & $\mathrm{O}(0 \%)$ & $0(0 \%)$ & $3(6,5 \%)$ & $43(93,5 \%)$ & $46(100 \%)$ \\
\hline
\end{tabular}

\section{Tableau 6}

Type d'équipement de protection collective mis en place dans les entreprises du secteur de la cosmétique.

Quels sont les équipements de protection collective et/ou matériel ergonomique dont disposent les locaux ? (plusieurs réponses possibles)

\begin{tabular}{ll}
\hline Siège adapté et réglable & $35(76,1 \%)$ \\
Bac à shampoing réglable & $32(69,6 \%)$ \\
Séparation des activités & $25(54,3 \%)$ \\
Système de ventilation & $21(45,7 \%)$ \\
efficace (VMC) & \\
Table de soin réglable & $15(32,6 \%)$ \\
Aspiration localisée & $8(17,4 \%)$ \\
Autres & $0(0 \%)$ \\
\hline
\end{tabular}

Un des médecins souligne l'importance du " matériel bien entretenu " dans la protection des salariées.

Les équipements de protection individuelle (EPI) mis à disposition des salariées en âge de procréer ou enceintes sont détaillés dans le Tableau 7.

Une question ouverte permettait aux médecins d'apporter des précisions sur d'éventuelles difficultés en rapport avec les EPI. L'un des médecins a indiqué un « changement du matériel à la demande " et un autre "jamais proposé par les employeurs ". Pour un médecin, la fréquence faible de port

Tableau 7

Fréquence de mise à disposition d'équipements de protection individuelle pour les salariées du secteur de la cosmétique en âge de procréer ou enceintes.

Quels sont les EPI mis à disposition de ces salariées en âge de procréer ou enceintes ? (plusieurs réponses possibles)

\begin{tabular}{lc}
\hline Gants & $41(89,1 \%)$ \\
Vêtements de travail spécifiques & $12(26,1 \%)$ \\
Masque & $7(15,2 \%)$ \\
Lunettes & $4(8,7 \%)$ \\
Chaussures & $3(6,5 \%)$ \\
Aucun & $2(4,3 \%)$ \\
Autres & $1(2,2 \%)^{a}$ \\
Charlotte & $0(0 \%)$
\end{tabular}

${ }^{a}$ Un médecin a précisé que des tabliers jetables étaient rarement proposés aux salariées. des EPI s'explique par la méconnaissance des risques par les salariées.

De nombreuses remarques ont été faites sur le port des gants (" changement de gants à chaque couleur ", " gants rarement portés ", " réutilisation des gants à usage unique après lavage et retournement ",...). Pour cinq médecins, la question de la nature des gants se pose (non adaptée au risque, gants en latex et non en nitrile ou vinyle). Un autre médecin rapporte une utilisation des gants en nitrile à usage unique pour les salariées des salons indépendants sur les recommandations du service de santé au travail alors que dans les enseignes nationales, les gants sont " collectifs, réutilisés, retournés et séchés ". Pour deux autres médecins, par contre, les enseignes nationales semblent mieux dotées en équipements de protection que les salons indépendants.

Quant aux formations, celles sur les TMS apparaissent plus fréquentes que celles sur le risque chimique (Tableau 8).

Un médecin précise que son service de santé au travail a proposé des formations sur ces deux thèmes mais qu'il a été impossible de les mettre en place, même sur le lieu de travail ("priorité des clients pour l'employeur").

La quatrième partie portait sur le rôle de l'équipe pluridisciplinaire en santé au travail.

Le premier thème abordé était celui des actions en milieu de travail et des actions d'information/sensibilisation.

Concernant les actions conduites par l'équipe pluridisciplinaire (Tableau 9), c'est la réalisation de la fiche d'entreprise qui est le plus souvent citée (89,1 \% des réponses), suivie par l'information et/ou sensibilisation lors des visites médicales $(84,8 \%)$ ou des entretiens infirmiers $(47,8 \%)$ et l'aide à l'évaluation des risques et conseils de prévention ( $78,3 \%$ ).

Un médecin précise qu'il remet des documents écrits lors de la visite médicale. Un autre a déjà fait intervenir un IPRP-toxicologue en onglerie.

L'information délivrée par l'équipe pluridisciplinaire consiste le plus souvent en une sensibilisation au port des EPI et/ou une sensibilisation des employeurs sur les risques professionnels et leurs obligations en termes de santé et sécurité au travail (Tableau 10).

Concernant les risques professionnels abordés par cette équipe pluridisciplinaire auprès des salariées du secteur de la cosmétique, les postures pénibles et le risque chimique ont 
Tableau 8

Mise en place de formations au bénéfice des salariées du secteur de la cosmétique.

\begin{tabular}{|c|c|c|c|c|}
\hline $\begin{array}{l}\text { Les salariées de la cosmétique bénéficient-elles de } \\
\text { formations concernant les risques suivants? }\end{array}$ & Oui & Non & NSPP/NSP & Total \\
\hline Formation " risque chimique " & $4(8,7 \%)$ & $19(41,3 \%)$ & $23(50 \%)$ & $46(100 \%)$ \\
\hline Formation " troubles musculosquelettiques" & $10(21,7 \%)$ & $18(39,1 \%)$ & $18(39,1 \%)$ & 46 (100\%) \\
\hline
\end{tabular}

Tableau 9

Pratique de l'équipe pluridisciplinaire dans la prévention des risques professionnels chez les salariées en âge de procréer ou enceintes travaillant dans le domaine de la cosmétique.

\begin{tabular}{ll}
$\begin{array}{l}\text { En quoi consiste la pratique de votre } \\
\text { équipe pluridisciplinaire dans la } \\
\text { prévention des risques professionnels } \\
\text { chez les salariées en âge de procréer } \\
\text { ou enceintes travaillant dans le } \\
\text { domaine cosmétique ? (plusieurs } \\
\text { réponses possibles) }\end{array}$ & $\begin{array}{l}\text { Nombre de } \\
\text { réponses (\%) }\end{array}$ \\
\hline $\begin{array}{l}\text { Réalisation de la fiche d'entreprise } \\
\text { Information et/ou sensibilisation } \\
\text { à l'occasion de visites médicales }\end{array}$ & $41(89,1 \%)$ \\
$\begin{array}{l}\text { Aide à l'évaluation des risques } \\
\text { et conseils de prévention }\end{array}$ & $39(84,8 \%)$ \\
$\begin{array}{l}\text { Information et/ou sensibilisation } \\
\text { à l'occasion d'entretiens } \\
\text { infirmiers en santé au travail }\end{array}$ & $36(78,3 \%)$ \\
$\begin{array}{l}\text { Organisation d'actions collectives } \\
\text { d'information et de sensibilisation }\end{array}$ & $22(47,8 \%)$ \\
$\begin{array}{l}\text { Réalisation de métrologies } \\
\text { Autres }\end{array}$ & $15(32,6 \%)$
\end{tabular}

\section{Tableau 10}

Thèmes sur lesquels porte l'information délivrée par l'équipe pluridisciplinaire.

\begin{tabular}{ll}
$\begin{array}{l}\text { Sur quels thèmes porte l'information } \\
\text { délivrée par l'équipe pluridisciplinaire ? } \\
\text { (plusieurs réponses possibles) }\end{array}$ & $\begin{array}{l}\text { Nombre de } \\
\text { réponses (\%) }\end{array}$ \\
\hline $\begin{array}{l}\text { Sensibilisation au port des EPI } \\
\text { Sensibilisation des employeurs } \\
\text { (risques professionnels et obligations) }\end{array}$ & $40(87 \%)$ \\
$\begin{array}{l}\text { Information des salariées } \\
\text { (risques professionnels }\end{array}$ & $40(87 \%)$ \\
$\quad$ et droits pendant la grossesse) & $22(47,8 \%)$ \\
$\begin{array}{l}\text { Information sur la nécessité } \\
\text { de déclarer une grossesse }\end{array}$ & $21(45,7 \%)$ \\
Autres & $0(0 \%)$ \\
\hline
\end{tabular}

été cochés par plus de $90 \%$ des médecins, et spécifiquement le risque CMR par plus de $50 \%$ (Tableau 11).

À propos du suivi en santé au travail, 21 médecins (45,7\%) ont répondu positivement à la question de la réalisation d'entretiens infirmiers pour les salariées du secteur de la cosmétique. Concernant les visites médicales (Tableau 12), 20 médecins $(43,5 \%)$ ont répondu n'être jamais sollicités par les salariées

\section{Tableau 11}

Importance de l'information délivrée par l'équipe pluridisciplinaire auprès des salariées du secteur de la cosmétique selon le risque professionnel.

Sur quels risques porte l'information

délivrée par l'équipe pluridisciplinaire

Nombre de

auprès des salariées du secteur de la

réponses (\%)

cosmétique ? (plusieurs réponses possibles)

Postures pénibles
Risque chimique
Risque CMR
Manutentions
Risque infectieux
Bruit
Stress
Chaleur
Vibrations
Aucun
Autres

$43(93,5 \%)$

$42(91,3 \%)$

$26(56,5 \%)$

$14(30,4 \%)$

$14(30,4 \%)$

$9(19,6 \%)$

$7(15,2 \%)$

$4(8,7 \%)$

$2(4,3 \%)$

$2(4,3 \%)$

$1(2,2 \%)^{a}$

${ }^{a}$ Un médecin cite le risque de coupure.

de ce secteur pour des visites à la demande en raison de leur grossesse. Quarante médecins $(87 \%)$ ont déclaré effectuer des visites de reprise après congé maternité mais seulement 26 $(56,6 \%)$ ont répondu les réaliser " toujours " ou " souvent ". Un médecin a précisé que les visites de reprise sont plus souvent demandées dans les enseignes nationales. Enfin, à la question « Les employeurs vous adressent-ils des salariées du domaine de la cosmétique pendant leur grossesse dans le cadre de la surveillance médicale renforcée ? ", 33 médecins $(71,7 \%)$ ont répondu que ce n'était jamais le cas.

Les dernières questions portaient sur les aménagements ou changements de poste et les arrêts de travail dans ce secteur de la cosmétique : 24 médecins (52,1\%) effectuent (toujours, souvent, parfois) des études de poste en vue d'aménagement pour les salariées enceintes et $35(76,1 \%)$ préconisent des aménagements pendant la grossesse (toujours pour 8 médecins, et souvent pour 9 médecins) (Tableau 13).

Ces aménagements peuvent être de plusieurs types (Tableau 14). Environ la moitié des médecins interrogés préconisent une limitation des postures pénibles et/ou du port de charges ; pour près de $40 \%$ des médecins, l'aménagement porte sur l'interdiction de manipulation de certains produits. Un médecin a souligné ici la notion d'inaptitude temporaire en cas d'aménagement impossible. 
Tableau 12

Sollicitations du médecin du travail par les salariées ou l'employeur dans le cadre du suivi de la grossesse au travail chez les salariées du secteur de la cosmétique.

\begin{tabular}{|c|c|c|c|c|c|c|}
\hline & Toujours & Souvent & Parfois & Jamais & Non concerné & Total \\
\hline $\begin{array}{l}\text { Visites à la demande des salariées en raison } \\
\text { de leur état de grossesse }\end{array}$ & o (० \%) & $1(2,2 \%)$ & $24(52,2 \%)$ & $20(43,5 \%)$ & $1(2,2 \%)$ & $46(100 \%)$ \\
\hline $\begin{array}{l}\text { Salariées enceintes adressées par l'employeur } \\
\text { dans le cadre de la surveillance médicale } \\
\text { renforcée }\end{array}$ & ० (० \%) & $1(2,2 \%)$ & $10(21,7 \%)$ & 33 (71,7 \%) & $2(4,3 \%)$ & $46(100 \%)$ \\
\hline $\begin{array}{l}\text { Salariées adressées par l'employeur en visite } \\
\text { de reprise après congé maternité }\end{array}$ & $9(19,6 \%)$ & 17 (37 \%) & $14(30,4 \%)$ & $5(10,9 \%)$ & $1(2,2 \%)$ & $46(100 \%)$ \\
\hline
\end{tabular}

\section{Tableau 13}

Études de poste et aménagements de poste dans les entreprises du secteur de la cosmétique pour les salariées enceintes exposées à des risques professionnels.

\begin{tabular}{|c|c|c|c|c|c|c|c|}
\hline & Toujours & Souvent & Parfois & Jamais & NSPP/NSP & Non concerné & Total \\
\hline $\begin{array}{l}\text { Études de poste effectuées par } \\
\text { le médecin du travail }\end{array}$ & $2(4,3 \%)$ & $3(6,5 \%)$ & $19(41,3 \%)$ & 16 (34,8 \%) & $2(4,3 \%)$ & $4(8,7 \%)$ & $46(100 \%)$ \\
\hline $\begin{array}{l}\text { Aménagements de poste } \\
\text { préconisés par le médecin } \\
\text { du travail }\end{array}$ & $8(17,4 \%)$ & 9 (19,6 \%) & $18(39,1 \%)$ & $5(10,9 \%)$ & $1(2,2 \%)$ & $5(10,9 \%)$ & $46(100 \%)$ \\
\hline
\end{tabular}

Tableau 14

Différents types d'aménagements de poste préconisés par le médecin du travail au bénéfice des salariées enceintes dans le secteur de la cosmétique.

Si vous préconisez des aménagements

de poste pendant la grossesse de

ces salariées, lesquels ? (plusieurs

réponses possibles)

Limitation des postures pénibles

Limitation du port de charges

Interdiction de manipulation

de certains produits

Aménagement d'horaires

Adaptation ou renforcement des EPI

Autres

\section{Nombre de réponses (\%) \\ $26(56,5 \%)$ \\ $22(47,8 \%)$ \\ $18(39,1 \%)$ \\ $16(34,8 \%)$ \\ $15(32,6 \%)$ \\ $1(2,2 \%)^{a}$}

${ }^{a}$ Un médecin rapporte l'achat de tabourets de coupe.

Quant à la notion d'arrêts de travail antérieurs à la date du congé légal de maternité, 32 médecins (39,1\%) ont déclaré en avoir connaissance dans le secteur de la cosmétique (Tableau 15).

Les changements temporaires d'affectation durant la grossesse pour nécessité médicale ou en raison de l'exposition à des risques particuliers tels que les reprotoxiques de catégorie $1 \mathrm{~A}$ ou $1 \mathrm{~B}$ sont rapportés par une minorité de médecins (Tableau 16).

Il leur était ensuite demandé l'origine de la demande de changement temporaire d'affectation pour nécessité médicale (article L.1225-7 du Code du travail) : l'employeur (2 réponses), la salariée ( 2 réponses) et/ou le médecin du travail (3 réponses). La même question a été posée sur l'origine de la demande de changement temporaire d'affectation pour l'exposition à des risques particuliers (article L. 1225-12) : l'employeur (1 réponse), la salariée (2 réponses) et/ou le médecin du travail (5 réponses).

Enfin, il était demandé aux médecins s'ils avaient connaissance, dans le secteur de la cosmétique, de la mise en œuvre des dispositions relatives à la suspension du contrat de travail avec garantie de rémunération, en cas d'impossibilité de reclassement, en rapport avec l'exposition à des risques particuliers (articles L. 1225-14 et R. 1225-4). Seuls 7 médecins $(15,2 \%)$ ont répondu positivement à cette question. Les différentes circonstances qu'ils ont rapportées sont : grossesse pathologique, inaptitude au poste et reclassement temporaire impossible ( 2 médecins), exposition aux produits CMR ou aux postures pénibles. L'un des médecins a précisé

Tableau 15

Connaissance par les médecins du travail d'arrêts de travail antérieurs à la date du congé légal de maternité.

\begin{tabular}{|c|c|c|c|c|c|c|c|}
\hline $\begin{array}{l}\text { Dans le secteur de la cosmétique, } \\
\text { avez-vous connaissance d'arrêts } \\
\text { de travail antérieurs à la date } \\
\text { du congé légal de maternité ? }\end{array}$ & Toujours & Souvent & Parfois & Jamais & NSPP/NSP & Non concerné & Total \\
\hline Nombre de réponses (\%) & o (o \%) & $6(13 \%)$ & $12(26,1 \%)$ & $14(30,4 \%)$ & $13(28,3 \%)$ & $1(2,2 \%)$ & $46(100 \%)$ \\
\hline
\end{tabular}


Tableau 16

Fréquence des changements temporaires d'affectation durant la grossesse selon la cause chez les salariées de la cosmétique.

\begin{tabular}{|c|c|c|c|c|}
\hline & Oui & Non & Non concerné & Total \\
\hline Pour nécessité médicale (article L. 1225-7 du Code du travail) & $3(6,5 \%)$ & $36(78,3 \%)$ & $7(15,2 \%)$ & $46(100 \%)$ \\
\hline Exposition à des risques particuliers (article L. 1225-12 du Code du travail) & $6(13 \%)$ & $35(76,1 \%)$ & $5(10,9 \%)$ & $46(100 \%)$ \\
\hline
\end{tabular}

qu'il a déjà utilisé ce dispositif deux fois en onglerie/esthétique ces dernières années en collaboration avec l'employeur (étude de poste et demande d'aménagement avec contreindication à la manipulation des huiles essentielles).

Pour finir, un seul médecin a eu connaissance de difficultés au retour au travail des salariées du secteur de la cosmétique, suite à leur congé maternité (difficultés en rapport avec l'allaitement et démission).

\section{Discussion}

L'objectif de cette enquête descriptive est de mieux connaître les pratiques, les ressources et les difficultés des médecins du travail, vis-à-vis du risque reprotoxique dans le secteur de la cosmétique. Dans la littérature, seule une étude sur la pratique des médecins du travail, mais concernant le risque cancérogène (prévention, dépistage et déclaration des cancers professionnels) a été retrouvée, basée sur des entretiens semidirectifs [21].

Pour la présente enquête, un auto-questionnaire ad hoc a été élaboré, dans l'objectif de recueillir le plus grand nombre possible de témoignages tout en limitant le temps de réponse. Ainsi, il n'était pas demandé aux médecins d'extraire des données à partir des dossiers médicaux. De plus, le choix a été fait d'un maximum de questions fermées avec une liste d'items proposés pour faciliter le remplissage, tout en laissant des champs libres via l'item "Autres " (avec possibilité de préciser) et quelques questions ouvertes.

Parmi les 257 médecins sollicités par mail via la SMST de la région PACA, environ $36 \%$ ont répondu à la première question (suivi de coiffeuses, esthéticiennes et/ou prothésistes ongulaires) et cette réponse était positive pour près de $18 \%$ des médecins. Ces derniers ont tous répondu à la suite du questionnaire, ce qui a permis d'obtenir 46 questionnaires exploitables. Le taux de réponse relativement faible peut s'expliquer par l'hypersollicitation des médecins du travail pour diverses études; de plus, la présentation réalisée lors d'une séance de la SMST pour sensibiliser les médecins, n'a pu toucher que les médecins présents. Par ailleurs, la liste de diffusion de la SMST ne nous a pas permis de cibler les médecins concernés par le secteur de la cosmétique. Ne connaissant pas l'effectif de ces derniers, l'évaluation du taux de réponse au sein de cette population cible est impossible. Nous ne pouvons donc pas conclure quant à la représentativité des médecins répondants, par rapport à la population globale des médecins du travail en charge du suivi des coiffeuses, esthéticiennes et/ou prothésistes ongulaires en région PACA. Cependant, les ratios rapportés par les médecins répondants, concernant la répartition des effectifs selon le type d'entreprise (salons indépendants à $80 \%$, enseignes nationales à $20 \%$ ) sont concordants avec ceux rapportés par la Fédération nationale de la coiffure $(90 \%$ de salons indépendants et $10 \%$ de salons franchisés) [22].

Dans notre enquête, huit médecins seulement (17,4 \%) ont déclaré avoir été confrontés à des questions en rapport avec la grossesse chez les salariées du secteur de la cosmétique. Ce chiffre semblant très bas, on peut se demander s'il est dû à une réelle absence de difficultés ou à l'absence de cas de grossesses portés à la connaissance du médecin du travail. En effet, des médecins n'ayant pas répondu positivement à cette question ont néanmoins souhaité préciser, à l'occasion d'une question ouverte, des problématiques particulièrement présentes chez les salariées de la coiffure et de l'esthétique.

Les médecins du travail ont ainsi mis en avant l'exposition aux produits cosmétiques, principal risque spécifique aux salariées de ce secteur.

Dans ce contexte, l'une des difficultés des médecins du travail réside dans le fait que les produits cosmétiques ne sont pas soumis au règlement européen CLP. Ils font, en effet, l'objet d'une réglementation spécifique et leurs effets sur la reproduction ne sont pas évalués, par les industriels ou les pouvoirs publics, vis-à-vis des utilisateurs professionnels. Ces spécificités et ces limites ont fait l'objet d'un dossier de l'INRS [22]. De plus, les fabricants de produits cosmétiques n'ont pas l'obligation de fournir des FDS. Néanmoins, selon le " règlement cosmétique " [23], les fabricants sont tenus d'évaluer les risques de leurs produits pour la santé des consommateurs dans les conditions normales d'utilisation en se basant sur les recommandations du Comité scientifique sur la sécurité des consommateurs (CSSC). Aucun contrôle du contenu ou de la qualité de cette évaluation n'est effectué avant la mise sur le marché. De plus, les tests vis-à-vis de la reproduction ne sont exigés que si une absorption importante par voie orale ou cutanée est attendue [22]. L'évaluation des risques ne prend pas en compte les conditions d'exposition pour les professionnels, malgré une fréquence d'utilisation plus importante que les consommateurs. Par ailleurs, même si les substances classées CMR sont interdites au public, des dérogations existent en cas d'avis favorable du CSSC [23].

Les fabricants ont néanmoins l'obligation d'indiquer la composition qualitative des produits cosmétiques (ingrédients par ordre décroissant de concentration) sur leur 
conditionnement. La nomenclature la plus fréquemment utilisée est l'International Nomenclature Cosmetic Ingredient (INCI) [22,24]. II est donc possible de retrouver le numéro CAS de chaque substance en consultant la base de données Coslng [25], puis de le rechercher dans d'autres bases de données toxicologiques comme les fiches DEMETER [20] de l'INRS ou la classification européenne CMR dont les dernières mises à jour sont accessibles sur le site de l'Agence européenne des produits chimiques [26]. Mais cette démarche, depuis l'inventaire des produits jusqu'à la recherche de leur éventuelle reprotoxicité, est longue et fastidieuse. Dans notre enquête, 35 médecins (76\%) ont répondu consulter la classification $C M R$, mais seuls 7 médecins (15,2\%) ont déclaré utiliser les fiches DEMETER.

Par ailleurs, même pour les produits pour lesquels les FDS sont obligatoires (produits d'entretien), celles-ci sont rarement fournies au médecin du travail par l'employeur comme le déplorent 35 médecins (76\%).

Chez les coiffeuses, les produits de coloration sont parmi les plus utilisés. Ils contiennent notamment des amines aromatiques, de l'ammoniac, de l'eau oxygénée, des diaminobenzènes, des diaminotoluènes ainsi que différents types de phénols. Les autres produits (shampoings, produits de décoloration, permanente, défrisage et frisage, laques...) sont également composés de persulfates, perborates, acide thioglycolique et ses sels, formaldéhyde, parabènes... $[9,10,27]$.

Certaines de ces substances peuvent être cancérogènes (formaldéhyde) et la plupart sont des allergènes cutanés ou respiratoires. Parmi les multiples substances contenues dans les produits utilisés dans ces métiers, il est possible que certaines soient classées reprotoxiques ou agissent comme perturbateurs endocriniens [22].

Les produits couramment utilisés par les prothésistes ongulaires contiennent de nombreux acrylates et méthacrylates et sont susceptibles de contenir des substances classées toxiques pour la reproduction comme le phtalate de dibutyle ( $\left.R_{1} B\right)$ ou le toluène ( $R 2)$. Le toluène fait d'ailleurs partie des cing substances pour lesquelles l'Agence nationale de sécurité sanitaire (ANSES) a formulé, en 2014, des recommandations visant à réduire l'exposition des femmes enceintes, notamment en milieu de travail [28]. D'autres substances, sans être classées, semblent entraîner des atteintes de la fertilité (éthanol) ou du développement (méthyléthylcétone) $[10,27]$.

Dans la méta-analyse de l'INRS [12], des excès de risque faibles, mais statistiquement significatifs (même si très faiblement supérieurs à la limite de la significativité), ont été retrouvés pour certains troubles de la reproduction chez les professions de la coiffure et des soins de beauté, sans que la part attribuable aux produits cosmétiques dans ces excès de risque puisse être estimée. Cependant, selon les auteurs, le lien avec les produits cosmétiques ne peut être écarté pour certains de ces troubles (délai nécessaire pour concevoir, faible poids de naissance, mortalité embryonnaire et fœtale). Certaines études incluent une caractérisation des expositions chimiques dans ces professions.

L'étude Pélagie, évaluant le risque pour le développement intra-utérin lié à l'exposition maternelle aux solvants pendant la grossesse, a révélé que 92,6 \% des coiffeuses se déclaraient exposées de façon régulière aux solvants et $100 \%$ étaient exposées selon la matrice emplois-exposition utilisée $[29,30]$.

Des études incluant une métrologie atmosphérique dans les salons de beauté, principalement de coiffure, ont également été publiées et concernent le formaldéhyde [31], les persulfates [32], l'éthanol [33,35], les acides thioglycolique et dithioglycolique [34], l'ammonium [34,35], l'isopropanolol, le toluène, le diaminotoluène et les phénylènediamines [35]. Elles ont mis en évidence des concentrations dans l'air intérieur relativement élevées mais inférieures aux valeurs limites lorsqu'elles sont déterminées.

Par ailleurs, il a été décrit que, même si les prothésistes ongulaires sont effectivement exposées aux solvants, les niveaux d'exposition mesurés sont limités et sans différence significative en fonction de la technique utilisée [36]. Aucune de ces études ne prend en compte l'exposition aux produits cosmétiques par voie cutanée.

Outre les valeurs limites d'exposition professionnelle (VLEP), il est à noter l'existence de valeurs toxicologiques de référence (VTR). À ce jour, l'ANSES a établi 43 VTR dont certaines sont fondées sur des effets reprotoxiques [37] comme le phtalate de dibutyle ou le toluène, utilisés par les prothésistes ongulaires notamment [38].

Dans notre enquête, les médecins du travail interrogés ont aussi souligné l'importance des contraintes posturales chez les salariées du secteur de la cosmétique. Chez les coiffeuses, la station debout prolongée avec piétinement ainsi que les gestes et postures défavorables sont prédominants dans la pratique professionnelle quotidienne [8]. En onglerie, il s'agit plutôt de gestes répétitifs et postures (membres supérieurs et rachis cervical) pouvant être associés à des vibrations transmises au système main-bras [9].

Quelques études ont été menées sur les postures pénibles [39] et la prévalence des TMS [40-43], notamment chez les coiffeuses.

Les douleurs articulaires liées au travail sont retrouvées plus fréquemment et de façon significative au niveau des cervicales, épaules, lombaires, poignets et membres inférieurs chez les salariées du secteur de la cosmétique [40,42,43]. Une augmentation statistiquement significative des TMS a également été mise en évidence, en rapport avec un travail physique ou une position inconfortable (membres supérieurs ou tronc) [41].

Les autres risques auxquels sont exposées les salariées du secteur de la cosmétique ainsi que des conseils de prévention 
sont détaillés dans des dossiers médicotechniques de l'INRS $[8,9]$.

L'évaluation des risques professionnels est une obligation de l'employeur et doit être consignée dans le DUER, mais $39 \%$ des médecins ont répondu que ce document n'était jamais mis en place dans les entreprises du secteur de la cosmétique dont ils assurent le suivi.

Quant aux éléments de prévention technique, les réponses des médecins font ainsi apparaître que de nombreuses salariées ne disposeraient pas de tous les équipements de protection, collectifs ou individuels, notamment par rapport à l'exposition aux produits cosmétiques. En effet, il est recommandé de séparer les activités en zones distinctes et d'associer un système de ventilation générale aux dispositifs de captage à la source [22]. Seuls 54,3\% des médecins ont coché l'item "Séparation des activités " et 45,7 \% l'item "Système de ventilation efficace " à la question sur les EPC. On peut également s'interroger sur l'efficacité de la séparation des activités puisqu'en pratique les zones distinctes restent relativement proches. Les gants, principal EPI dans ce secteur, semblent être fréquemment utilisés (selon 89 \% des médecins) même si quelques perfectionnements sont possibles.

La formation des salariées du secteur de la cosmétique concernant le risque chimique et les TMS semble, quant à elle, peu mise en place (4 et 10 médecins respectivement).

L'équipe pluridisciplinaire en santé au travail a également un rôle à jouer dans la formation et l'information des salariées. Selon les résultats, $47,8 \%$ des médecins ont déclaré que l'information délivrée aux salariées par l'équipe pluridisciplinaire porte sur les risques professionnels et leurs droits au travail pendant la grossesse.

Il est à noter que les professionnels de santé eux-mêmes, dont les médecins du travail, sont peu formés sur les risques professionnels pour la grossesse. La formation des professionnels constitue d'ailleurs un axe d'amélioration de la prévention des risques selon l'avis d'experts de l'INRS [19]. Par ailleurs, les données recueillies montrent que 41,3\% des médecins du travail disent n'échanger avec aucun autre acteur de prévention et 93,5\% ne jamais avoir été en contact avec la sage-femme, acteur pourtant important du suivi de grossesse ayant également un rôle dans l'information préventive des femmes enceintes. En revanche, ils sont, pour la plupart, en lien avec le médecin généraliste $(80,4 \%)$ et/ou le gynécologue $(76,1 \%)$. Ce travail en interdisciplinarité pourrait donc être amélioré, notamment lors de la grossesse.

Le médecin du travail est un interlocuteur privilégié concernant l'information des femmes sur la réglementation relative à la grossesse au travail et les mesures préventives [44]. Mais, $58,7 \%$ des médecins ont répondu ne jamais être informés, par l'employeur, des déclarations de grossesse. Par ailleurs, pour $71,7 \%$ des médecins, l'employeur ne leur adresse jamais une femme enceinte de ce secteur dans le cadre de la surveillance médicale renforcée ; 52,2 \% des médecins disent qu'une visite à la demande est parfois sollicitée par les salariées ellesmêmes. En dehors de problématiques spécifiques, le médecin du travail donc semble être peu informé et peu sollicité pour le suivi de ces femmes enceintes. Ils le sont peu également pour les visites de reprise après congé maternité, pourtant obligatoires, $41 \%$ des médecins déclarant que ces visites sont peu ou pas demandées par les employeurs.

La prévention des risques professionnels pour la femme enceinte repose pourtant sur la nécessaire sensibilisation des femmes en âge de procréer à déclarer leur état de grossesse le plus précocement possible, pour que des mesures préventives adaptées, si elles sont nécessaires, puissent être prises rapidement [16]. Le médecin du travail a un rôle important à jouer dans cette sensibilisation, mais, parmi les thèmes proposés sur lesquels était susceptible de porter l'information délivrée par l'équipe pluridisciplinaire, seuls 45,7\% des médecins ont coché " la nécessité de déclarer précocement une grossesse, voire un désir de grossesse ".

Une grande majorité de médecins $(76,1 \%)$ ont répondu préconiser (toujours, souvent ou parfois) des aménagements de poste au bénéfice des femmes enceintes de ce secteur, portant sur la limitation de contraintes physiques (postures pénibles, port de charges), l'interdiction de manipulation de certains produits, des aménagements d'horaires ou encore le renforcement des EPI. Mais il s'agissait d'une liste de propositions à cocher et la question portait sur les aménagements préconisés par le médecin du travail et non sur ceux finalement mis en œuvre par l'employeur. En pratique, ces aménagements ne sont pas toujours réalisés; quatre médecins ont d'ailleurs souligné, dans des questions à réponses ouvertes, le recours à l'inaptitude temporaire au poste de travail en cas d'aménagement impossible.

Rares sont les médecins rapportant avoir connaissance de changement temporaire d'affectation pour nécessité médicale ( 3 médecins) ou en raison de l'exposition à des risques particuliers (6 médecins) dans le secteur de la cosmétique comme cela est prévu dans les articles L. 1225-7 et L.1225-12 du Code du travail. Les dispositions relatives à la suspension du contrat de travail avec garantie de rémunération en rapport avec l'exposition à des risques particuliers (articles L. 122514 et R. 1225-4 du Code du travail) et leurs conditions strictes de mise en œuvre (décret $\mathrm{n}^{\circ}$ 2002-1282 du 23 octobre 2002, circulaire DSS/4 C/DRT/CT $3 \mathrm{n}^{\circ}$ 99-72 du 8 février 1999) semblent également être peu connues puisqu'un seul témoignage a été recueilli. Pour information, un formulaire de déclaration " Incompatibilité du travail avec la grossesse ", pour demander le versement de l'allocation journalière de maternité, est disponible sur le site ameli.fr [45].

En pratique, alors que des aménagements de poste auraient peut-être permis la poursuite d'une activité professionnelle, de nombreuses salariées présentent des arrêts de travail antérieurs à la date du congé légal de maternité ; cela est rapporté par 39,1 \% des médecins du travail de notre 
échantillon, mais ils ne sont pas nécessairement au courant de ces arrêts. Cette pratique semble être beaucoup plus fréquente, $78,5 \%$ des femmes enceintes ayant eu recours à ces arrêts de travail précoces en 2010, d'après les données de I'ENP [1].

\section{Conclusion}

Le secteur de la cosmétique, très majoritairement féminin, expose donc naturellement à la question des risques pour la grossesse. Le rôle du médecin du travail est important chez les salariées enceintes ou en âge de procréer. Son action débute bien avant la grossesse par son rôle de conseiller pour l'évaluation des risques et l'information des femmes en âge de procréer. L'évaluation des risques implique d'identifier les différents facteurs de risque susceptibles d'avoir un impact sur le déroulement de la grossesse, ce qui permet de mettre en place des mesures de prévention (dont la priorité à la substitution d'éventuels produits (MR) et de définir en amont des conduites à tenir (aménagement/changement de poste) en cas de survenue d'une grossesse parmi les salariées. L'information des femmes en âge de procréer doit porter sur les risques professionnels, les moyens de prévention, la nécessité de déclarer sa grossesse le plus précocement possible et les dispositifs légaux de protection de la femme enceinte au travail. Ces éléments pourraient être intégrés dès la formation initiale des professionnelles du secteur de la cosmétique.

Il semble également nécessaire d'améliorer la veille du déroulement des grossesses en milieu professionnel en créant un groupe de référence et un réseau d'experts pour le développement de la prévention des risques comme le préconise l'avis d'experts de l'INRS [19].

Par ailleurs, même si de nombreux dangers potentiels sont identifiés, les connaissances sur les risques des expositions professionnelles pour la fertilité et la grossesse sont encore limitées. Cela est particulièrement vrai dans le secteur de l'esthétique, devant la multiplicité des produits cosmétiques, leur réglementation spécifique, le manque de données sur la caractérisation des expositions professionnelles et sur l'évaluation des risques en termes de troubles de la reproduction.

\section{Financement}

Ce travail a bénéficié d'une aide du gouvernement français, gérée par l'Agence nationale de la recherche au titre du projet «Investissements d'Avenir A*MIDEX " portant la référence $n^{\circ}$ ANR-11-IDEX-0001-02.

\section{Remerciements}

Les auteurs remercient le Dr Danielle Charrier et le Dr Florence Bajon, respectivement présidente et secrétaire de la Société de médecine et santé du travail, d'ergonomie et de toxicologie de la région PACA, pour leur soutien dans la réalisation de ce travail. Ils remercient tous les médecins du travail ayant participé à l'enquête.
Déclaration de liens d'intérêts

Les auteurs déclarent ne pas avoir de liens d'intérêts.

\section{Références}

[1] Enquête Nationale Périnatale 2010. Les naissances en 2010 et leur évolution depuis 2003. [Internet. Consulté 22 sept 2016]. Disponible sur : http://social-sante.gouv.fr/IMG/pdf/ Les_naissances_en_2010_et_leur_evolution_depuis_2003.pdf.

[2] Risques professionnels par métiers. Enquête SUMER 2010. [Internet].[Consulté 22 sept 2016]. Disponible sur : http:// travail-emploi.gouv.fr/IMG/pdf/Synthese_Stat_no_05_-_Risques_ professionnels_par_metiers.pdf.

[3] Meron M, Okba M, Viney X. Les femmes et les métiers : vingt ans d'évolutions contrastées. Données Soc INSEE; 2006.

[4] Fiche médico-professionnelle Coiffeur [Internet. Consulté 22 sept 2016]. Disponible sur : http://www.fmpcisme.org/ FMPPDF/22092016022054--annexe.pdf.

[5] Fiche métier Esthéticienne [Internet. Consulté 22 sept 2016]. Disponible sur : http://www.bossons-fute.fr/index.php?option= com content\&view=article\&id=302:ficheo074\&catid $=2 \&$ Itemid $=3$.

[6] Fiche médico-professionnelle Prothésiste ongulaire [Internet. Consulté 22 sept 2016]. Disponible sur : http://www.fmpcisme. org/FMPPDF/22092016055857-_annexe.pdf.

[7] Eeckelaert L, Dontas S, Georgiadou E, et al. Occupational health and safety in the hairdressing sector. Luxemb Luxemb Eur Agency Saf Health Work 2014;1-30.

[8] Bruneteau A, Bechmann L, Picot P, et al. Évaluation et prévention des risques dans les salons de coiffure. DMT INRS 2004;99:315-66.

[9] Beaumont D, Collard C, Cortin C. Évaluation et prévention des risques chez les prothésistes ongulaires. Dossier médico-technique TC117. Doc Med Trav 2008;113:21-36.

[10] Fiche d'entreprise Salon de coiffure [Internet. Consulté 22 sept 2016]. Disponible sur : http://www.bossons-fute.fr/index. php?option=com_content\&view=article\&id $=$ 659:entrepriseoo $17 \&$ catid $=15 \&$ Itemid $=7$.

[11] Testud F, Abadia-Benoist G. Risques professionnels chez la femme enceinte. EMC 16-66o-A-10; 2010.

[12] Picot C, Bouslama M, Collot-Fertey D, et al. État des connaissances sur les liens entre les troubles de la reproduction et l'exposition aux produits cosmétiques chez les professionnels de la coiffure et des soins de beauté. Approche épidémiologique et méta-analytique; 2013.

[13] Henrotin J-B, Picot C, Bouslama M, et al. Reproductive disorders in hairdressers and cosmetologists: a meta-analytical approach. J Occup Health 2015;57(6):485-96.

[14] Kim D, Kang M-Y, Choi S, et al. Reproductive disorders among cosmetologists and hairdressers: a meta-analysis. Int Arch Occup Environ Health 2016;89:739-53.

[15] Produits chimiques cancérogènes, mutagènes, toxiques pour la reproduction. Classification réglementaire [Internet. Consulté 22 sept 2016]. Disponible sur : http://www.inrs.fr/dms/inrs/ CataloguePapier/ED/TI-ED-976/ed976.pdf.

[16] Conso F, Contassot JC, Falcy M, et al. Salariées enceintes exposées à des substances toxiques pour le développement fœtal. Surveillance médicale. Doc Med Trav 2005;101:9-21.

[17] Recommandations professionnelles. Comment mieux informer les femmes enceintes ? [Internet. Consulté 22 sept 2016]. Disponible sur : http://www.has-sante.fr/portail/upload/ docs/application/pdf/femmes_enceintes_recos.pdf. 
[18] Recommandations professionnelles. Suivi et orientation des femmes enceintes en fonction des situations à risque identifiées. [Internet. Consulté 22 sept 2016]. Disponible sur : http:// www.has-sante.fr/portail/upload/docs/application/pdf/ suivi_des_femmes_enceintes_-_recommandations_23-04-2008. pdf.

[19] Lafon D. Grossesse et travail. Quels sont les risques pour l'enfant à naître ?. EDP Science; 2010, 562 p. [Avis d'expert].

[20] Fiches Demeter [Internet. Consulté 22 sept 2016]. Disponible sur : http://www.inrs.fr/publications/bdd/demeter.html.

[21] Pardon C, Dumesnil H, Charrier D, et al. Médecins du travail et cancers professionnels: attitudes, opinions et pratiques. Une recherche qualitative dans le Sud-Est de la France. Rev Epidemiol Sante Publ 2009;57(6):456-65.

[22] Lafon D, Abou-Anoma G, Bouslama M, et al. Exposition aux produits cosmétiques et risques pour la grossesse chez les professionnelles de la coiffure. Ref Sante Trav 2014;(138):23-45.

[23] Règlement CE no 1223/2009 du Parlement européen et du Conseil du 30 novembre 2009 relatif aux produits cosmétiques [Internet. Consulté 1 oct 2016]. Disponible sur : http://eurlex.europa.eu/LexUriServ/LexUriServ.do?uri=OJ:L:2009:342:0059: 0209:fr:pdf.

[24] Règlementation des produits cosmétiques [Internet. Consulté 22 sept 2016]. Disponible sur : http://ansm.sante.fr/var/ansm site/storage/original/application/6733575728ba78afo829d411 02651e82.pdf.

[25] CosIng. European Commission [Internet. Consulté 22 sept 2016]. Disponible sur : http://ec.europa.eu/growth/ tools-databases/cosing/index.cfm?fuseaction=search.simple.

[26] European Chemicals Agency ECHA [Internet]. European Chemicals Agency ECHA. [Consulté 22 sept 2016]. Disponible sur : https://echa.europa.eu/fr.

[27] Pak VM, Powers M, Liu J. Occupational chemical exposures among cosmetologists. Workplace Health Saf 2013;61(12):522-9.

[28] L'Anses publie ses recommandations en vue de réduire l'exposition à cinq substances reprotoxiques et/ou perturbatrices endocriniennes, Anses - Agence nationale de sécurité sanitaire de l'alimentation, de l'environnement et du travail [Internet. consulté 2 nov 2016]. Disponible sur: https://www.anses.fr/fr/ content/lanses-publie-ses-recommandations-en-vue-de-r\%C3 \% Agduire-lexposition-\%C3 \%Ao-cinq-substance.

[29] Garlantézec R, Monfort C, Rouget F, et al. Exposition aux solvants pendant la grossesse : étude Pélagie. Arch Mal Prof Environ 2008;69(2):272-5.

[30] Ferrario F, Continenza D, Pisani P, et al. In: Hogstedt C, Reuterwall C, editors. Description of a job exposure matrix for sixteen agents which are or may be related to respiratory cancer. Prog Occup Epidemiol. Elsevier Sci Publ BV; 1988 p. 379-82.
[31] Tsigonia A, Lagoudi A, Chandrinou S, et al. Indoor air in beauty salons and occupational health exposure of cosmetologists to chemical substances. Int J Environ Res Publ Health 2010;7(1):314-24.

[32] Nilsson PT, Marini S, Wierzbicka A, et al. Characterization of hairdresser exposure to airborne particles during hair bleaching. Ann Occup Hyg 2016;60(1):90-100.

[33] Muiswinkel W-J van, Kromhout H, Onos T, et al. Monitoring and modelling of exposure to ethanol in hairdressing salons. Ann Occup Hyg 1997;41(2):235-47.

[34] Oikawa D, Takeuchi W, Murata S, et al. Measurement of concentrations of thioglycolic acid, dithiodiglycolic acid and ammonia in indoor air of a beauty salon. J Occup Health 2012;54(5):370-5.

[35] Elihollund B, Moen BE. Chemical exposure in hairdresser salons: effect of local exhaust ventilation. Ann Occup Hyg 1998;42(4):277-81.

[36] Gjølstad M, Thorud S, Molander P. Occupational exposure to airborne solvents during nail sculpturing. J Environ Monit 2006;8(5):537-42.

[37] Liste des Valeurs Toxicologiques de Référence (VTR) construites par l'Anses. [Internet. Consulté 22 sept 2016]. Disponible sur : https://www.anses.fr/fr/content/liste-des-valeurs-toxicologiquesde-r\%C3 \%Agf\%C3 \%Agrence-vtr-construites-par-1\%E2 \%80 \% 99anses.

[38] Valeurs toxicologiques de référence (VTR). Guide d'élaboration de VTR. Rapport d'expertise collective. [Internet. Consulté 22 sept 2016]. Disponible sur: https://www.anses.fr/fr/ system/files/VTR2012SA0275Ra.pdf.

[39] Wahlström J, Mathiassen SE, Liv P, et al. Upper arm postures and movements in female hairdressers across four full working days. Ann Occup Hyg 2010;54(5):584-94.

[40] Tsigonia A, Tanagra D, Linos A, et al. Musculoskeletal disorders among cosmetologists. Int J Environ Res Public Health 2009; 6(12):2967-79.

[41] Mussi G, Gouveia N. Prevalence of work-related musculoskeletal disorders in Brazilian hairdressers. Occup Med 2008;58(5):367-9.

[42] Bradshaw L, Harris-Roberts J, Bowen J, et al. Self-reported workrelated symptoms in hairdressers. Occup Med 2011;61(5):328-34.

[43] Aweto HA, Tella BA, Johnson OY. Prevalence of work-related musculoskeletal disorders among hairdressers. Int J Occup Med Environ Health 2015;28(3):545-55.

[44] Salariées en état de grossesse. Aide-mémoire juridique. [Internet. Consulté 22 sept 2016]. Disponible sur : http://www.inrs. $\mathrm{fr} / \mathrm{dms} / \mathrm{inrs} /$ CataloguePapier/ED/TI-TJ-14/tj14.pdf.

[45] Formulaire_incompatibilite_travail_grossesse.pdf [Internet. consulté 31 oct 2016]. Disponible sur : http://www.ameli.fr/ fileadmin/user_upload/documents/Formulaire_incompatibilite travail_grossesse.pdf. 